\title{
Mixed Sub-Fractional Brownian Motion
}

\author{
Mounir Zili
}

\begin{abstract}
A new extension of the sub-fractional Brownian motion, and thus of the Brownian motion, is introduced. It is a linear combination of a finite number of sub-fractional Brownian motions, that we have chosen to call the mixed sub-fractional Brownian motion. In this paper, we study some basic properties of this process, its non-Markovian and non-stationarity characteristics, the conditions under which it is a semimartingale, and the main features of its sample paths. We also show that this process could serve to get a good model of certain phenomena, taking not only the sign (like in the case of the sub-fractional Brownian motion), but also the strength of dependence between the increments of this phenomena into account.
\end{abstract}

Keywords Mixed Gaussian processes, sub-fractional Brownian motion, Hausdorff dimension.

Mathematics Subject Classification 60G15; 60G17; 60G20, 28A80.

\section{Introduction}

The sub-fractional Brownian motion ( $\mathrm{sfBm}$ ) is an extension of a Brownian motion $(\mathrm{Bm})$, which was investigated in many papers (e.g. [4, 21]). It is a stochastic process $\xi^{H}=\left\{\xi_{t}^{H} ; t \geq 0\right\}$, defined on a probability space $(\Omega, F, \mathbb{P})$ by:

$$
\forall t \in \mathbb{R}_{+}, \quad \xi_{t}^{H}=\frac{B_{t}^{H}+B_{-t}^{H}}{\sqrt{2}},
$$

where $H \in] 0,1\left[\right.$, and $\left\{B^{H}(t), t \in \mathbb{R}\right\}$ is a fractional Brownian motion (fBm) on the whole real line; i.e. $B^{H}$ is a continuous and centered Gaussian process with covariance function

$$
\operatorname{cov}\left(B^{H}(t), B^{H}(s)\right)=1 / 2\left(|t|^{2 H}+|s|^{2 H}-|t-s|^{2 H}\right) .
$$

The index $H$ is called the Hurst parameter of $B^{H}$. In some applications (such as turbulence) $\mathrm{fBm}$ is an adequate model for small increments, but it seems to be inadequate for large increments (For more information about fBm, see for example [13]). For this reason, $\xi^{H}$ may be an alternative to $\mathrm{fBm}$ in some stochastic models. Moreover, the sfBm arises from occupation time fluctuations of branching particle systems with Poisson initial condition [4].

In this paper, an extension of the sfBm is introduced; it will be called the mixed sub-fractional Brownian motion (msfBm). More precisely, for $N \in \mathbb{N} \backslash\{0\}$, 
$\left.H=\left(H_{1}, H_{2}, \ldots, H_{N}\right) \in\right] 0,1\left[^{N}\right.$ and $a=\left(a_{1}, a_{2}, \ldots, a_{N}\right) \in \mathbb{R}^{N} \backslash\{(0, \ldots, 0)\}$, the mixed sub-fractional Brownian motion (msfBm) of parameters $N, a$ and $H$, is the process $S=\left\{S_{t}^{H}(N, a) ; t \geq 0\right\}=\left\{S_{t}^{H} ; t \geq 0\right\}$, defined on the probability space $(\Omega, F, \mathbb{P})$ by:

$$
\forall t \in \mathbb{R}_{+}, \quad S_{t}^{H}(N, a)=\sum_{i=1}^{N} a_{i} \xi^{H_{i}}(t)
$$

where $\left(\xi^{H_{i}}\right)_{i \in\{1, \ldots, N\}}$ is a family of independent sub-fractional Brownian motions of Hurst parameters $H_{i}$ defined on $(\Omega, F, \mathbb{P})$.

If $N=1$, and $a_{1}=1, \quad S^{H}=\xi^{H}$ is a sub-fractional Brownian motion, and if $N=1, H_{1}=1 / 2$ and $a_{1}=1, \quad S^{H}$ is a standard Brownian motion. So, the $\mathrm{msfBm}$ is more general, and this is a first reason which returns this process interesting to be investigated.

In 8 , the authors studied on one hand some key properties of the particular process $S^{\left(1 / 2, H_{2}\right)}(2, a)$ with $\left.H_{2} \in\right] 0,1\left[\right.$ and $a=\left(a_{1}, a_{2}\right) \in \mathbb{R}^{2} \backslash\{(0,0)\}$, and on the other hand its martingale properties.

This paper is concerned with the study of the msfBm in general. Our first objective is to extend the properties obtained in 8 to the general case. Particularly we show that by a suitable choice of the parameters $a$ and $H$, the process $S^{H}$ could serve to get a good model of certain phenomena, taking not only the sign (as in the case of the fBm and the sfBm), but also the strength of dependence between the increments of the phenomena into account; and this is another main motivation to investigate this process.

Our second aim is to study some analytic and geometric properties of the sample paths of $S^{H}$. Especially, we investigate the Hausdorff dimensions of its graph, range and level sets.

The rest of this paper is organised as follows. Section 2 contains basic properties of the msfBm, in particular the non-stationnarity, the mixed-selfsimilarity 1 and the non-Markovian properties.

In section 3 , we investigate some msfBm increments properties. We especially explicit the covariances of the $\mathrm{msfBm}$ increments on non-overlapping intervals, and we analyze them to understand "how far" the msfBm is from a process with stationary increments.

Section 4 is concerned with analytic and geometric properties of the sample paths of $S^{H}$. We show the Hölder-continuity and the non-differentiability of the tajectories, and we give the Hausdorff dimensions of the graph, range and level sets. The methods used in our proofs are inspired by the papers [23, [24] and 2], and are based particularly on the Frostman's theorem and the capacity argument (see [12]).

In the last section, we investigate the semimartingale property, according to the values of the parameters $H$ and $a$. More precisely, we show that the process $S^{H}(N, a)$ is a semimartingale, if and only if it exists $k_{0} \in\{1, . ., N\} ; H_{k_{0}}=1 / 2$, $a_{k_{0}} \neq 0$ and for every $\left.i \in\{1, \ldots, N\} \backslash\left\{k_{0}\right\}, H_{i} \in\{1 / 2\} \cup\right] 3 / 4,1[$.

\footnotetext{
${ }^{1}$ The mixed-self-similarity property was introduced by M. Zili in [26]
} 


\section{The main properties}

Let us first recall some properties of the sfBm (see [4] and 21] for proofs and for further information).

Lemma 1 The sfBm $\left(\xi_{t}^{H}\right)_{t \in \mathbb{R}_{+}}$satisfies the following properties:

- $\xi^{H}$ is a centered Gaussian process.

- $\forall s \in \mathbb{R}_{+}, \forall t \in \mathbb{R}_{+}$,

$$
\left.\operatorname{Cov}\left(\xi_{t}^{H}, \xi_{s}^{H}\right)\right)=s^{2 H}+t^{2 H}-1 / 2\left((s+t)^{2 H}+|t-s|^{2 H}\right) .
$$

- For all $(s, t) \in \mathbb{R}_{+}^{2} ; s \leq t$,

$$
E\left(\xi_{t}^{H}-\xi_{s}^{H}\right)^{2}=-2^{2 H-1}\left(t^{2 H}+s^{2 H}\right)+(t+s)^{2 H}+(t-s)^{2 H} .
$$

- The increments of the smfBm are not stationary.

As a first obvious consequence of Lemma 1 we state:

Lemma 2 The msfBm satisfies the following properties:

1. $S_{t}^{H}$ is a centered Gaussian process.

2. $\forall s \in \mathbb{R}_{+}, \forall t \in \mathbb{R}_{+}$,

$$
\operatorname{Cov}\left(S_{t}^{H}(a), S_{s}^{H}(a)\right)=\sum_{i=1}^{N} a_{i}^{2}\left[t^{2 H_{i}}+s^{2 H_{i}}-1 / 2\left[(s+t)^{2 H_{i}}+|t-s|^{2 H_{i}}\right]\right] .
$$

3.

$$
\forall t \in \mathbb{R}_{+}, \quad \mathbb{E}\left(\left(S_{t}^{H}(a)\right)^{2}\right)=\sum_{i=1}^{N} a_{i}^{2}\left[\left(2-2^{2 H_{i}-1}\right) t^{2 H_{i}}\right] .
$$

Let us now study the mixed-self-similarity property (see [26]) of the msfBm.

Lemma 3 For any $h>0$, the processes $\left\{S_{h t}^{H}(a)\right\}$ and $\left\{S_{t}^{H}\left(a_{1} h^{H_{1}}, a_{2} h^{H_{2}}, \ldots, a_{N} h^{H_{N}}\right)\right\}$ have the same law.

Proof. It is due to the fact that for fixed $h>0$, the processes $\left\{S_{h t}^{H}(a)\right\}$ and $\left\{S_{t}^{H}\left(a_{1} h^{H_{1}}, a_{2} h^{H_{2}}, \ldots, a_{N} h^{H_{N}}\right)\right\}$ are Gaussian, centered and have the same covariance function.

The following lemma deals with the non-Markovian property of the msfBm. 
Lemma 4 For every $a=\left(a_{1}, \ldots, a_{N}\right) \in \mathbb{R}^{N}$ and $\left.H=\left(H_{1}, \ldots, H_{N}\right) \in\right] 0 ; 1\left[\left[^{N}\right.\right.$ such that it exists $j \in\{1, \ldots, N\} ; a_{j} \neq 0$ and $H_{j} \neq 1 / 2,\left(S_{t}^{H}(a)\right)_{t \in \mathbb{R}_{+}}$is not a Markovian process.

Proof. The process $S^{H}$ is a centered Gaussian. Moreover, it exists $j \in$ $\{1, \ldots, N\} ; a_{j} \neq 0$ and $H_{j} \neq 1 / 2$. Therefore, for all $t>0$,

$$
\operatorname{Cov}\left(S_{t}^{H}, S_{t}^{H}\right)=\sum_{i=1}^{N} a_{i}^{2}\left(\left(2-2^{2 H_{i}-1}\right)\right) t^{2 H_{i}}>0 .
$$

If $S^{H}$ were a Markovian process, according to [16], for all $s<t<u$ we would have:

$$
\operatorname{Cov}\left(S_{s}^{H}, S_{u}^{H}\right) \operatorname{Cov}\left(S_{t}^{H}, S_{t}^{H}\right)=\operatorname{Cov}\left(S_{s}^{H}, S_{t}^{H}\right) \operatorname{Cov}\left(S_{t}^{H}, S_{u}^{H}\right) .
$$

Let us consider

$H_{i_{0}}=\min \left\{H_{i} ; i \in\{1, \ldots, N\} ; a_{i} \neq 0\right\}$ and $H_{i_{1}}=\max \left\{H_{i} ; i \in\{1, \ldots, N\} ; a_{i} \neq 0\right\}$.

First case: If $H_{j}>1 / 2$. In this case, $H_{i_{1}}>1 / 2$. By expression (6) and equation (8) with

$$
1<s=\sqrt{t}<t<u=t^{2}
$$

we would have,

$$
\begin{aligned}
& \sum_{i=1}^{N} a_{i}^{2}\left(2-2^{2 H_{i}-1}\right) t^{2 H_{i}} \\
& \times \sum_{i=1}^{N} a_{i}^{2}\left(t^{4 H_{i}}+t^{H_{i}}-1 / 2 t^{4 H_{i}}\left[\left(1+t^{-3 / 2}\right)^{2 H_{i}}+\left(1-t^{-3 / 2}\right)^{2 H_{i}}\right]\right) \\
= & \sum_{i=1}^{N} a_{i}^{2}\left(t^{H_{i}}+t^{2 H_{i}}-1 / 2 t^{2 H_{i}}\left[\left(1+t^{-1 / 2}\right)^{2 H_{i}}+\left(1-t^{-1 / 2}\right)^{2 H_{i}}\right]\right) \\
& \times \sum_{i=1}^{N} a_{i}^{2}\left(t^{2 H_{i}}+t^{4 H_{i}}-1 / 2 t^{4 H_{i}}\left[\left(1+t^{-1}\right)^{2 H_{i}}+\left(1-t^{-1}\right)^{2 H_{i}}\right]\right),
\end{aligned}
$$

Since, when $h \rightarrow 0$ we have

$$
(1+h)^{2 H_{i}}+(1-h)^{2 H_{i}}=2+2 H_{i}\left(2 H_{i}-1\right) h^{2}+o\left(h^{2}\right),
$$

equations (9) and (10) imply that for $t \rightarrow \infty$, 


$$
\begin{aligned}
& \sum_{i=1}^{N} a_{i}^{2}\left(2-2^{2 H_{i}-1}\right) t^{2 H_{i}} \times \sum_{i=1}^{N} a_{i}^{2}\left(t^{H_{i}}-H_{i}\left(2 H_{i}-1\right) t^{4 H_{i}-3}+o\left(t^{4 H_{i}-3}\right)\right) \\
- & {\left[\sum_{i=1}^{N} a_{i}^{2}\left(t^{H_{i}}-H_{i}\left(2 H_{i}-1\right) t^{2 H_{i}-1}+o\left(t^{2 H_{i}-1}\right)\right)\right.} \\
& \left.\times \sum_{i=1}^{N} a_{i}^{2}\left(t^{2 H_{i}}-H_{i}\left(2 H_{i}-1\right) t^{4 H_{i}-2}+o\left(t^{4 H_{i}-2}\right)\right)\right] \\
= & 0 .
\end{aligned}
$$

The left member of equation (11) would tend to zero as t goes to infty; consequently, we would have

$$
\lim _{t \rightarrow \infty} a_{i_{1}}^{4}\left(1-2^{2 H_{i_{1}}-1}\right) t^{3 H_{i_{1}}}=0,
$$

which is true if, and only if $H_{i_{1}}=1 / 2$. So $S^{H}$ is not a Markovian process.

Second case: If $H_{j}<1 / 2$. In this case, $H_{i_{0}}<1 / 2$. Using the same technique as explained in the first case, but with

$$
0<s=t^{2}<t<u=\sqrt{t}<1 \text { and } t \rightarrow 0
$$

we get

$$
\lim _{t \rightarrow 0} a_{i_{0}}^{4}\left(1-2^{2 H_{i_{0}}-1}\right) t^{6 H_{i_{0}}}=0,
$$

which is true if, and only if $H_{i_{0}}=1 / 2$. So in this case too, $S^{H}$ is not a Markovian process.

\section{$3 \quad$ Study of the msfBm increments}

In the following propostion, we will characterize the increments second moment of the msfBm.

Proposition 5 For all $(s, t) \in \mathbb{R}_{+}^{2} ; s \leq t$,

1.

$$
\begin{aligned}
& E\left(S_{t}^{H}(a)-S_{s}^{H}(a)\right)^{2} \\
= & \sum_{i=1}^{N} a_{i}^{2}\left(-2^{2 H_{i}-1}\left(t^{2 H_{i}}+s^{2 H_{i}}\right)+(t+s)^{2 H_{i}}+(t-s)^{2 H_{i}}\right) .
\end{aligned}
$$


2.

$$
\sum_{i=1}^{N} a_{i}^{2} \gamma_{i}(t-s)^{2 H_{i}} \leq E\left(S_{t}^{H}(a)-S_{s}^{H}(a)\right)^{2} \leq \sum_{i=1}^{N} a_{i}^{2} \nu_{i}(t-s)^{2 H_{i}}
$$

where

$$
\gamma_{i}=\left\{\begin{array}{rll}
2-2^{2 H_{i}-1} & \text { if } & H_{i}>1 / 2 \\
1 & \text { if } & H_{i} \leq 1 / 2
\end{array}\right.
$$

and

$$
\nu_{i}=\left\{\begin{array}{r}
1 \quad \text { if } \quad H_{i}>1 / 2 \\
2-2^{2 H-1} \quad \text { if } \quad H_{i} \leq 1 / 2
\end{array}\right.
$$

Proof. The first result is due to equation (5) and to the fact that the processes $\xi^{H_{i}}$ are independent. By the same fact, the second assertion is a direct consequence of part (3) of the Theorem on p. 407 of [4].

Remark: From proposition 5, it is clear that the msfBm does not have stationary increments, but this property is replaced by inequalities (15).

In the following Lemma, by an easy calculus and by equation (6), we explicit the covariances of the msfBm increments on non-overlapping intervals.

Lemma 6 If for $0 \leq u<v \leq s<t$, we denote by

$$
C_{u, v, s, t}=C_{u, v, s, t}(a)=\operatorname{Cov}\left(S_{v}^{H}(a)-S_{u}^{H}(a), S_{t}^{H}(a)-S_{s}^{H}(a)\right)
$$

then,

$$
\begin{aligned}
C_{u, v, s, t} & =\sum_{i=1}^{N} \frac{a_{i}^{2}}{2}\left[(t+u)^{2 H_{i}}+(t-u)^{2 H_{i}}+(s+v)^{2 H_{i}}+(s-v)^{2 H_{i}}\right. \\
& \left.-(t+v)^{2 H_{i}}-(t-v)^{2 H_{i}}-(s+u)^{2 H_{i}}-(s-u)^{2 H_{i}}\right] .
\end{aligned}
$$

As a first consequence of Lemma 6. we can specify the sign of correlation between the increments of the msfBm, according to the values of $H$.

Corollary 7 For $0 \leq u<v \leq s<t$,

1. $C_{u, v, s, t}=0$, if for every $i \in\{1, \ldots, N\}, H_{i}=1 / 2$,

2. $C_{u, v, s, t}>0$, if for every $i \in\{1, \ldots, N\}, H_{i}>1 / 2$,

3. $C_{u, v, s, t}<0$, if for every $i \in\{1, \ldots, N\}, H_{i}<1 / 2$. 
Proof. The first assertion is trivial. To check the second one, let us write

$$
C_{u, v, s, t}=\sum_{i=1}^{N} \frac{a_{i}^{2}}{2}\left(g_{i}(t)-g_{i}(s)\right)
$$

where

$$
g_{i}(t)=-(t+v)^{2 H_{i}}-(t-v)^{2 H_{i}}+(t+u)^{2 H_{i}}+(t-u)^{2 H_{i}} .
$$

For every $i \in\{1, \ldots, N\}$, the function $g_{i}$ is differentiable and for every $t>0$,

$$
g_{i}^{\prime}(t)=2 H_{i}\left(-(t+v)^{2 H_{i}-1}-(t-v)^{2 H_{i}-1}+(t+u)^{2 H_{i}-1}+(t-u)^{2 H_{i}-1}\right) .
$$

Since $H_{i}>1 / 2$, the function $x \longmapsto x^{2 H_{i}-1}$ is concave and $g_{i}^{\prime}(t)>0$. Consequently, $g_{i}$ increases and the second assertion holds.

The third result proof is similar.

As a second consequence of Lemma 6, it is easy to get the following main result:

Corollary 8 For $0 \leq u<v \leq s<t, i \in\{1, \ldots, N\}$ and $\left(a_{j}\right)_{j \in\{1, \ldots, N\} \backslash\{i\}}$, if $b$ and $c$ are two real numbers such that; $|b| \leq|c|$, then

$$
\begin{gathered}
C_{u, v, s, t}\left(a_{1}, \ldots, a_{i-1}, b, a_{i+1}, \ldots, a_{N}\right)<C_{u, v, s, t}\left(a_{1}, \ldots, a_{i-1}, c, a_{i+1}, \ldots, a_{N}\right) \\
{[\text { respectively }=,>] \text { if } H_{i}<1 / 2\left[\text { respectively } H_{i}=1 / 2, \quad H_{i}>1 / 2\right] .}
\end{gathered}
$$

So, from Corollary 8 we see that, for $0 \leq u<v \leq s<t, i \in\{1, \ldots, N\}$ and $\left(a_{j}\right)_{j \in\{1, \ldots, N\} \backslash\{i\}}$, if $H_{i}>1 / 2\left[\right.$ respectively $\left.H_{i}<1 / 2\right]$,

- the smaller $[$ larger $]|b|$ is, the stronger the dependence between the increments of $S^{H}\left(\left(a_{1}, \ldots, a_{i-1}, b, a_{i+1}, \ldots, a_{N}\right)\right.$ is,

- the larger $[$ smaller $]|b|$ is, the weaker the dependence between the increments of $S^{H}\left(\left(a_{1}, \ldots, a_{i-1}, b, a_{i+1}, \ldots, a_{N}\right)\right.$ is.

Consequence: In the modelling of a certain phenomena, we can choose $H=\left(H_{1}, \ldots, H_{N}\right)$ and $a=\left(a_{1}, \ldots, a_{N}\right)$ suitably in a such manner that $\left\{S_{t}^{H}(a)\right\}$ permits to obtain a good model, taking not only the sign (as in the case of $\mathrm{fBm}$ and $\mathrm{sfBm}$ ), but also the strength of dependence between the increments of the phenomena into account.

In the following Lemma, we prove that the increments of the msfBm are short-range dependent. For this, let us denote, for every nonnegative real $x$ and nonnegative integer $n$,

$$
C(x, n)=C_{x, x+1, x+n, x+n+1}=\operatorname{Cov}\left(S_{x+1}^{H}-S_{x}^{H}, S_{x+n+1}^{H}-S_{x+n}^{H}\right) .
$$

By Lemma 6, a Taylor expansion and an easy calculus we get: 
Lemma 9 1. For every nonnegative integers $p$ and $n$ such that $n \geq 1$, we have

$$
\begin{aligned}
C(p, n)= & \sum_{i=1}^{N} \frac{a_{i}^{2}}{2}\left[(n+1)^{2 H_{i}}-2 n^{2 H_{i}}+(n-1)^{2 H_{i}}\right. \\
& \left.-(2 p+n+2)^{2 H_{i}}+2(2 p+n+1)^{2 H_{i}}-(2 p+n)^{2 H_{i}}\right] .
\end{aligned}
$$

2. For every $H \in] 0,1\left[^{N}\right.$ and $p \in \mathbb{N}$, it holds that as $n \rightarrow \infty$,

$$
C(p, n)=\sum_{i=1}^{N}\left[2\left(1-H_{i}\right) H_{i}\left(2 H_{i}-1\right)(2 p+1) a_{i}^{2} n^{2 H_{i}-3}+o\left(n^{2 H_{i}-3}\right)\right] .
$$

3. For every $H \in] 0,1\left[^{N}\right.$ and every $p \in \mathbb{N}$,

$$
\sum_{n \geq 0} C(p, n)<\infty
$$

We can now analyze the function $x \longmapsto C(x, n)$ to understand "how far" the $\mathrm{msfBm}$ is from a process with stationary increments. Let us first consider, for $\left.N \in \mathbb{N} \backslash\{0\}, H=\left(H_{1}, H_{2}, \ldots, H_{N}\right) \in\right] 0,1\left[{ }^{N}\right.$ and $a=\left(a_{1}, a_{2}, \ldots, a_{N}\right) \in \mathbb{R}^{N} \backslash$ $\{(0, \ldots, 0)\}$, the process, $M^{H}=\left\{M_{t}^{H}(N, a) ; t \geq 0\right\}$, defined on the probability space $(\Omega, F, \mathbb{P})$ by:

$$
\forall t \in \mathbb{R}_{+}, \quad M_{t}^{H}(N, a)=\sum_{i=1}^{N} a_{i} B_{t}^{H_{i}}
$$

where the $B_{t}^{H_{i}}$ 's are independent fractional Brownian motion with Hurst parameters $H_{i}$ defined on $(\Omega, F, \mathbb{P})$.

This process is called the mixed fractional Brownian motion $(\mathrm{mfBm})$. It has been introduced in 2001 by Cheridito [5], studied in 2007 by M.Zili [26] in the particular case where $N=2$ and $H_{1}=1 / 2$, then extended in 2008, by Y.MiaoW.Ren-Z.Ren [14 to the case where $N=2$ and $\left.H_{1} \in\right] 0,1[$. In 2009, further remarks on the generalized form (21) of $M^{H}$ have been given by C. Thäle [20].

The following Lemma is due to [26]:

Lemma 10 If for every positive real $x$, we denote by

$$
R(x, n)=R_{x, x+1, x+n, x+n+1}=\operatorname{Cov}\left(M_{x+1}^{H}-M_{x}^{H}, M_{x+n+1}^{H}-M_{x+n}^{H}\right),
$$

then

$$
R(x, n)=R(0, n)=\sum_{i=1}^{N} \frac{a_{i}^{2}}{2}\left[(n+1)^{2 H_{i}}-2 n^{2 H_{i}}+(n-1)^{2 H_{i}}\right] .
$$


From Lemma 10 it is obvious that the increments of the mfBm are stationnary. So, to meet our target, it suffices to analyze the function $x \longmapsto C(x, n)$ to understand "how far" the msfBm is from the $\mathrm{mfBm}$.

Lemma 11 For every $n$ it holds that as $x \rightarrow \infty$,

$$
C(x, n)=R(0, n)-\sum_{i=1}^{N} a_{i}^{2} 2^{2 H_{i}-1} H_{i}\left(2 H_{i}-1\right) x^{2\left(H_{i}-1\right)}+o\left(x^{2\left(H_{i}-1\right)}\right),
$$

therefore $\lim _{x \rightarrow \infty} C(x, n)=R(0, n)$ for every $n$.

Proof. By Lemma 9 Lemma 10 and a Taylor expansion, we get the result.

\section{Study of the msfBm sample paths}

In the sequel of the paper, we denote by $i_{0}$ an integer such that

$$
i_{0} \in\{1, \ldots, N\} \text { and } H_{i_{0}}=\min \left\{H_{i} ; i \in\{1, \ldots, N\} \text { and } a_{i} \neq 0\right\} .
$$

In the following lemma, we check the continuity of the msfBm sample paths, and we even show that the parameter $H_{i_{0}}$, and consequently $H$, controls their regularity.

Lemma 12 For all $T>0$ and $\gamma<H_{i_{0}}$, the msfBm has a modification which sample-paths have a Hölder-continuity, with order $\gamma$, on the interval $[0 ; T]$.

Proof. By [4, for every $i \in\{1, \ldots, N\}$, if $\left.H_{i} \in\right] 0,1\left[\right.$ and $0<\epsilon<H_{i}$, there exists a nonnegative random variable $G_{H_{i}, \epsilon, T}$ such that $E\left(G_{H_{i}, \epsilon, T}^{p}\right)<\infty$ for every $p \geq 1$, and

$$
\left|\xi^{H_{i}}(t)-\xi^{H_{i}}(s)\right| \leq G_{H_{i}, \epsilon, T}|t-s|^{H_{i}-\epsilon} \quad \text { a.s },
$$

for all $s, t \in[0, T]$.

So, for every $0<\epsilon<H_{i_{0}}$, and $s, t \in[0 ; T]$

$$
\left|S_{t}^{H}-S_{s}^{H}\right|=\left|\sum_{i=1}^{N} a_{i}\left(\xi^{H_{i}}(t)-\xi^{H_{i}}(s)\right)\right| \leq G_{\epsilon, T}|t-s|^{H_{i_{0}}-\epsilon} \quad \text { a.s. }
$$

where

$$
G_{\epsilon, T}=\sum_{i=1}^{N}\left|a_{i}\right| G_{H_{i}, \epsilon, T} T^{H_{i}-H_{i_{0}}}
$$

for which we have clearly, $E\left(G_{\epsilon, T}^{p}\right)<\infty$ for every $p \geq 1$. And the proof is finished.

In the next Lemma, we prove that with probability one, the msfBm sample paths are not differentiable. 
Lemma 13 For every $H \in] 0 ; 1\left[^{N}\right.$,

$$
\lim _{\epsilon \rightarrow 0^{+}} \sup _{t \in\left[t_{0}-\epsilon, t_{0}+\epsilon\right]}\left|\frac{S^{H}(t)-S^{H}\left(t_{0}\right)}{t-t_{0}}\right|=+\infty,
$$

with probability one for every $t_{0} \in \mathbb{R}$.

Proof. . If for $m \in \mathbb{N}$, we denote $A^{(m)}=\cap_{n=1}^{+\infty} A_{n}^{(m)}$ where, for $n \in \mathbb{N}$,

$$
A_{n}^{(m)}=\left\{w \in \Omega ; \sup _{t \in\left[t_{0}-\frac{1}{n}, t_{0}+\frac{1}{n}\right]}\left|\frac{S^{H}(t)-S^{H}\left(t_{0}\right)}{t-t_{0}}\right|>m\right\},
$$

to obtain the result it is enough to prove that $P\left(\cap_{m=1}^{+\infty} A^{(m)}\right)=1$.

On one hand, the fact that each of the sequences $\left(A_{n}^{(m)}\right)_{n}$ and $\left(A^{(m)}\right)_{m}$ decreases, allows to write

$$
P\left(\cap_{m=1}^{+\infty} A^{(m)}\right)=\lim _{m \rightarrow+\infty} P\left(A^{(m)}\right)=\lim _{m \rightarrow+\infty} \lim _{n \rightarrow+\infty} P\left(A_{n}^{(m)}\right) .
$$

On another hand,

$$
P\left(A_{n}^{(m)}\right) \geq P\left(\left|S^{H}\left(t_{0}+\frac{1}{n}\right)-S^{H}\left(t_{0}\right)\right|>\frac{m}{n}\right) .
$$

So to prove the lemma, it is enough to show that

$$
\forall m \in \mathbb{N}, \lim _{n \rightarrow+\infty} P\left(\left|S^{H}\left(t_{0}+\frac{1}{n}\right)-S^{H}\left(t_{0}\right)\right| \leq \frac{m}{n}\right)=0 .
$$

Since $S^{H}\left(t_{0}+\frac{1}{n}\right)-S^{H}\left(t_{0}\right)$ is a centered Gaussian random variable with variance

$$
\sigma_{n}^{2}\left(t_{0}\right)=E\left(S^{H}\left(t_{0}+\frac{1}{n}\right)-S^{H}\left(t_{0}\right)\right)^{2},
$$

we have:

$$
\begin{aligned}
P\left(\left|S^{H}\left(t_{0}+\frac{1}{n}\right)-S^{H}\left(t_{0}\right)\right| \leq \frac{m}{n}\right) & =\frac{1}{\sigma_{n}\left(t_{0}\right) \sqrt{2 \pi}} \int_{-\frac{m}{n}}^{\frac{m}{n}} \exp \left(-\frac{x^{2}}{2 \sigma_{n}^{2}\left(t_{0}\right)}\right) d x \\
& \leq 2 \frac{m}{n} \times \frac{1}{\sigma_{n}\left(t_{0}\right) \sqrt{2 \pi}} .
\end{aligned}
$$

From Proposition 5 ,

$$
\sigma_{n}^{2}\left(t_{0}\right)=\sum_{i=1}^{N} a_{i}^{2}\left(-2^{2 H_{i}-1}\left(\left(t_{0}+\frac{1}{n}\right)^{2 H_{i}}+t_{0}^{2 H_{i}}\right)+\left(2 t_{0}+\frac{1}{n}\right)^{2 H_{i}}+\frac{1}{n^{2 H_{i}}}\right),
$$


then, for large $n, \sigma_{n}^{2}\left(t_{0}\right) \approx \sum_{i=1}^{N} a_{i}^{2} n^{-2 H_{i}}$.

$$
\begin{gathered}
\text { So } \lim _{n \rightarrow+\infty} n^{2} \sigma_{n}^{2}\left(t_{0}\right)=\lim _{n \rightarrow+\infty} \sum_{i=1}^{N} a_{i}^{2} n^{2-2 H_{i}}=+\infty \text {, and consequently } \\
\lim _{n \rightarrow+\infty} P\left(\left|S^{H}\left(t_{0}+\frac{1}{n}\right)-S^{H}\left(t_{0}\right)\right| \leq \frac{m}{n}\right)=0 .
\end{gathered}
$$

Denoting the range of the restriction of $S^{H}(a)$ on $[0, T]$ by

$$
S^{H}([0, T])=\left\{S^{H}(t) ; t \in[0, T]\right\},
$$

its graph by

$$
\operatorname{Grf}_{T} S^{H}(a)=\left\{\left(t, S_{t}^{H}(a)\right) ; t \in[0, T]\right\},
$$

the graph of $S^{H}(a)$ by

$$
\operatorname{Grf} S^{H}(a)=\left\{\left(t, S_{t}^{H}(a)\right) ; t \in[0,+\infty[\},\right.
$$

and the level set of the restriction of $S^{H}(a)$ on $[\epsilon, T]$ by

$$
L_{x}^{\epsilon}=\left\{t \in[\epsilon, T] ; S^{H}(t)=x\right\},
$$

where $T>\epsilon>0$, the aim of the sequel of this section, is to study the Hausdorff dimensions of the sets defined in (27), (28), (29) and (30).

Let us first recall briefly the definition of Hausdorff dimension. For each $\alpha>0, E \subset \mathbb{R}^{d}$, the $\alpha$ - dimensional Hausdorff measure of $E$ is defined by

$$
M^{\alpha}(E)=\lim _{\delta \rightarrow 0} \inf \left\{|E|^{\alpha} ; E \subset \cup_{k=1}^{\infty} E_{k} ;\left|E_{k}\right|<\delta\right\},
$$

where $\left|E_{k}\right|$ is the diameter of the set $E_{k}$ and the infinimum is taken over all coverings $\left(E_{k}\right)_{k \in \mathbb{N}}$ of $E$. The Hausdorff dimension of $E$ is defined by

$$
\operatorname{dim} E=\inf \left\{\alpha>0 ; M^{\alpha}(E)=0\right\}=\sup \left\{\alpha>0 ; M^{\alpha}(E)=+\infty\right\} .
$$

Lemma 14 The Hausdorff dimension of the graph of $S^{H}(a)$ equals $2-H_{i_{0}}$ with probability 1, where $H_{i_{0}}$ is defined by (25).

Proof. By Lemma 12, for all $T>0$, the msfBm has a modification which sample-paths have a Hölder-continuity, with order $\gamma<H_{i_{0}}$ on the interval $[0 ; T]$. So, according to [23], for all $T>0$, with probability 1 ,

$$
\operatorname{dimGrf}_{T} S^{H}(a) \leq 2-H_{i_{0}},
$$


which implies that

$$
\operatorname{dim} \operatorname{GrfS}^{H}(a) \leq 2-H_{i_{0}} .
$$

Now, thanks to the Frostman's Theorem (see e.g. 9]) to obtain the second inequality we only need to show that for every $T>0$, the occupation measure $\nu$ of $t \longmapsto\left(t, S_{t}^{H}(a)\right)$, when $t$ is restricted to the interval [0;T], has, with probability 1 , a finite $u$-dimensional energy, for any $u \in] 1,2-H_{i_{0}}[$. More precisely, for any Borel set $A \subset \mathbb{R}^{2}, \nu(A)$ is defined as the integral

$$
\nu(A)=\int_{0}^{T} \mathbf{1}_{\left\{\left(t, S_{t}^{H}(a)\right) \in A\right\}} d t,
$$

where, for every set $U \subset \mathbb{R}^{2}, \mathbf{1}_{U}$ denotes the characteristic function of the set $U$, and we need to prove that with probability 1 the integral

$$
\int_{G r f_{T} S^{H}(a)} \int_{G r f_{T} S^{H}(a)}|x-y|^{-u} \nu(d x) \nu(d y)
$$

is finite. By a monotone class argument this is easily seen to be equivalent to

$$
\int_{0}^{T} \int_{0}^{T}\left(|s-t|+\left|S_{s}^{H}(a)-S_{t}^{H}(a)\right|\right)^{-u} d s d t<+\infty,
$$

which follows from

$$
\int_{0}^{T} \int_{0}^{T} \mathbb{E}\left(\left(|s-t|+\left|S_{s}^{H}(a)-S_{t}^{H}(a)\right|\right)^{-u}\right) d s d t<+\infty .
$$

In order to get (36), we need the following preliminary lemma.

Lemma 15 For all $(s, t) \in \mathbb{R} \times \mathbb{R}, s \neq t$ and for every real $u>1$, we have

$$
\mathbb{E}\left(\left(|s-t|+\left|S_{t}^{H}(a)-S_{s}^{H}(a)\right|\right)^{-u}\right) \leq c|t-s|^{1-u} \sigma^{-1}(s, t),
$$

where

$$
\sigma^{2}(s, t)=\mathbb{E}\left(S_{t}^{H}(a)-S_{s}^{H}(a)\right)^{2}
$$

and $c>0$ is a constant.

Proof. (of Lemma 15) We have 


$$
\begin{aligned}
& \mathbb{E}\left(\left(|s-t|+\left|S_{t}^{H}(a)-S_{s}^{H}(a)\right|\right)^{-u}\right) \\
= & \frac{1}{\sigma(s, t) \sqrt{2 \pi}} \int_{\mathbb{R}}(|t-s|+|x|)^{-u} \exp \left(-\frac{x^{2}}{2 \sigma^{2}(s, t)}\right) d x \\
= & \frac{2}{\sigma(s, t) \sqrt{2 \pi}} \int_{0}^{|t-s|}(|t-s|+|x|)^{-u} \exp \left(-\frac{x^{2}}{2 \sigma^{2}(s, t)}\right) d x \\
& +\frac{2}{\sigma(s, t) \sqrt{2 \pi}} \int_{|t-s|}^{+\infty}(|t-s|+|x|)^{-u} \exp \left(-\frac{x^{2}}{2 \sigma^{2}(s, t)}\right) d x \\
\leq & \frac{2}{\sigma(s, t) \sqrt{2 \pi}}\left(\int_{0}^{|t-s|}|t-s|^{-u} d x+\int_{|t-s|}^{+\infty}|x|^{-u} d x\right) \\
\leq & \frac{2}{\sigma(s, t) \sqrt{2 \pi}}\left(|t-s|^{1-u}+\frac{1}{u-1}|t-s|^{1-u}\right) \\
\leq & c|t-s|^{1-u} \sigma^{-1}(s, t),
\end{aligned}
$$

with

$$
c=\frac{\sqrt{2}}{\sqrt{\pi}} \frac{u}{u-1} .
$$

Let us now prove (36). By Lemma 15 then by Proposition 5 we get:

$$
\begin{aligned}
& \int_{0}^{T} \int_{0}^{T} \mathbb{E}\left(\left(|s-t|+\left|S_{s}^{H}(a)-S_{t}^{H}(a)\right|\right)^{-u}\right) d s d t \\
\leq & \int_{0}^{T} \int_{0}^{T} c|t-s|^{1-u} \sigma^{-1}(s, t) d s d t \\
\leq & \int_{0}^{T} \int_{0}^{T} \frac{c}{a_{i_{0}} \sqrt{\gamma_{i_{0}}}}|t-s|^{1-u-H_{i_{0}}} d s d t
\end{aligned}
$$

where $\gamma_{i_{0}}$ is defined by (16). Since $\left.u \in\right] 1,2-H_{i_{0}}[$, it is easy to check that

$$
\int_{0}^{T} \int_{0}^{T}|t-s|^{1-u-H_{i_{0}}} d s d t<+\infty
$$

which achieves the proof.

Lemma 16 The Hausdorff dimension of the range $S^{H}([0, T])$ equals 1 with probability 1. 
Proof. We have clearly $\operatorname{dim} S^{H}([0, T]) \leq 1$ a.s., so we only need to prove that

$$
1 \leq \operatorname{dim} S^{H}([0, T]) \text { a.s. }
$$

Note that for $\epsilon \in] 0, T[$,

$$
\operatorname{dim} S^{H}([0, T]) \geq \operatorname{dim} S^{H}([\epsilon, T]),
$$

and that for any standard normal variable $X$ and $0<\gamma<1$, we have

$$
E\left(|X|^{-\gamma}\right)<\infty .
$$

Hence by Frostman's theorem (see e.g. 99), it is sufficient to show that for all $0<\gamma<1$,

$$
E_{\gamma}=\int_{\epsilon}^{T} \int_{\epsilon}^{T} E\left(\left|S^{H}(s)-S^{H}(t)\right|^{-\gamma}\right) d s d t<+\infty .
$$

From Proposition 5 we see that there exist positive and finite constants $c_{1}$ and $c_{2}$ such that for all $s, t \in[0, T]$,

$$
c_{1}|s-t|^{2 H_{i_{0}}} \leq E\left(\left(S^{H}(s)-S^{H}(t)\right)^{2}\right) \leq c_{2}|s-t|^{2 H_{i_{0}}} .
$$

So by (41) and (42), it exists a positive and finite constant $c_{3}$ such that

$$
E_{\gamma} \leq c_{3} \int_{\epsilon}^{T} \int_{\epsilon}^{T}|s-t|^{-\gamma H_{i_{0}}} d s d t
$$

Since $0<\gamma H_{i_{0}}<1$, the second member of the inequality (43) is finite and we get the result.

The following lemma is necessary for the study of the Hausdorff dimension of the level set $L_{x}^{\epsilon}$.

Lemma 17 If we denote by $\operatorname{Var}(Y \mid Z)$ the conditional variance of $Y$ given $Z$, there exists a constant $c>0$ such that for all $s, t \in I$,

$$
\operatorname{Var}\left(S^{H}(t) \mid S^{H}(s)\right) \geq c|s-t|^{2 H_{i_{0}}} .
$$

Proof. Since the conditional variance in (44) is the square of the $L^{2}(\mathbb{P})$ distance of $S^{H}(t)$ from the subspace generated by $S^{H}(s)$, we have

$$
\operatorname{Var}\left(S^{H}(t) \mid S^{H}(s)\right)=\inf _{b \in \mathbb{R}} \mathbb{E}\left(S^{H}(t)-b S^{H}(s)\right)^{2} .
$$

So, from the definition (3) of the msfBm, and from the independence of the sfBm's $\xi^{H_{i}}$ we can write 


$$
\begin{aligned}
\operatorname{Var}\left(S^{H}(t) \mid S^{H}(s)\right) & =\inf _{b \in \mathbb{R}} \sum_{i=1}^{N} \mathbb{E}\left(\xi^{H_{i}}(t)-b \xi^{H_{i}}(s)\right)^{2} \\
& \geq \inf _{b \in \mathbb{R}} \mathbb{E}\left(\xi^{H_{i_{0}}}(t)-b \xi^{H_{i_{0}}}(s)\right)^{2} \\
& =\operatorname{Var}\left(\xi^{H_{i_{0}}}(t) \mid \xi^{H_{i_{0}}}(s)\right) .
\end{aligned}
$$

Thanks to Yan and Shen [Theorem 2.1, [25], we know that it exists a constant $c>0$ such that

$$
\operatorname{Var}\left(\xi^{H_{i_{0}}}(t) \mid \xi^{H_{i_{0}}}(s)\right) \geq c|t-s|^{2 H_{i_{0}}} .
$$

Equations (46) and (47) complete the proof.

Lemma 18 For every $x \in \mathbb{R}$, and $0<\epsilon<T$, with positive probability

$$
\operatorname{dim}_{H}\left(L_{x}^{\epsilon}\right)=1-H_{i_{0}}
$$

where $H_{i_{0}}$ is defined by (25).

Proof. For an integer $n \geq 1$, devide the interval $[\epsilon, T]$ into $m_{n}$ sub-intervals $I_{n, l}$ of length $n^{-1 / H_{i_{0}}}$. Then

$$
m_{n} \leq T \times n^{1 / H_{i_{0}}} .
$$

Let $0<\delta<1$ be fixed and let $\tau_{n, l}=\epsilon+l n^{-1 / H_{i_{0}}}$. By Lemma 3.3 of 22 and Lemma 2.2 of 18 we get:

$$
\begin{aligned}
\mathbf{P}\left\{x \in S^{H}\left(I_{n, l}\right)\right\} \leq & \mathbf{P}\left\{\max _{s, t \in I_{n, l}}\left|S^{H}(s)-S^{H}(t)\right| \leq n^{-(1-\delta)} ; x \in S^{H}\left(I_{n, l}\right)\right\} \\
& +\mathbf{P}\left\{\max _{s, t \in I_{n, l}}\left|S^{H}(s)-S^{H}(t)\right|>n^{-(1-\delta)}\right\} \\
\leq & \mathbf{P}\left\{\left|S^{H}\left(\tau_{n, l}\right)-x\right| \leq n^{-(1-\delta)}\right\}+\exp \left(-c_{1} n^{2 \delta}\right) \\
\leq & c_{2} n^{-(1-\delta)} .
\end{aligned}
$$

Define a covering $\left\{I_{n, l}^{\prime}\right\}$ of $L_{x}$ by $I_{n, l}^{\prime}=I_{n, l}$ if $x \in S^{H}\left(I_{n, l}\right)$ and $I_{n, l}^{\prime}=\emptyset$ otherwise. Denote the number of such sets $\left\{I_{n, l}^{\prime}\right\}$ by $M_{n}$. By (49) and (50) we get:

$$
\begin{aligned}
E\left(M_{n}\right) & \leq E\left(m_{n} \times \mathbf{1}_{\left\{x \in S^{H}\left(I_{n, l}\right)\right\}}\right) \\
& \leq T \times n^{1 / H_{i_{0}}} \times \mathbb{P}\left\{x \in S^{H}\left(I_{n, l}\right)\right\} \\
& \leq c_{3} \times n^{1 / H_{i_{0}}-1+\delta}
\end{aligned}
$$


where $c_{3}$ is a positive constant.

Let $\eta=1 / H_{i_{0}}-(1-2 \delta)$. We consider the sequence of integers $n_{i}=2^{i}(i \geq 1)$. Then by (51), the Markov inequality and the Borel-Cantelli lemma we see that almost surely $M_{n_{i}} \leq c_{4} n_{i}^{\eta}$ for all $i$ large enough. This implies that $\operatorname{dim}_{H} L_{x}^{\epsilon} \leq$ $H_{i_{0}} \eta$ almost surely. Letting $\delta \downarrow 0$ along rational numbers, we get

$$
\operatorname{dim}_{H} L_{x}^{\epsilon} \leq 1-H_{i_{0}} \text { a.s. }
$$

To prove the lower bound for $\operatorname{dim}_{H} L_{x}^{\epsilon}$ in (48), we consider $\delta>0$ a small constant such that

$$
\gamma=1-H_{i_{0}}(1+\delta)>0
$$

Note that if we can prove that there is a constant $c_{5}>0$, independent of $\delta$, such that

$$
\mathbf{P}\left\{\operatorname{dim}_{H} L_{x}^{\epsilon} \geq \gamma\right\} \geq c_{5},
$$

then the lower bound in (48) will follow by letting $\delta \downarrow 0$.

Our proof of (54) is based on the capacity argument due to Kahane [see [12]]. Similar methods have been used in [1], 2], [19] and [23].

Let $\mathcal{M}_{\gamma}^{+}$be the space of all non-negative measures on $\mathbb{R}$ with finite $\gamma-$ energy. It is known [cf. [1] that $\mathcal{M}_{\gamma}^{+}$is a complete metric space under the metric

$$
\|\mu\|_{\gamma}=\int_{\mathbb{R}} \int_{\mathbb{R}} \frac{\mu(d t) \mu(d s)}{|t-s|^{\gamma}} .
$$

We define a sequence of random positive measures $\mu_{n}:=\mu_{n}(x,$.$) on the$ Borel sets $C$ of $[\epsilon, T]$ by

$$
\begin{aligned}
\mu_{n}(C) & =\int_{C} \sqrt{2 \pi} \exp \left(-\left(S^{H}(t)-x\right)^{2} / 2\right) d t \\
& =\int_{C} \int_{\mathbb{R}} \exp \left(-\xi^{2} / 2+i \xi\left(S^{H}(t)-x\right)\right) d \xi d t .
\end{aligned}
$$

It follows from [12] (p. 206) or [19] (p.17) that if there exist positive and finite constants $c_{6}, c_{7}$ and $c_{8}$ such that

$$
\begin{gathered}
\mathbb{E}\left(\left\|\mu_{n}\right\|\right) \geq c_{6}, \quad \mathbb{E}\left(\left\|\mu_{n}\right\|^{2}\right) \leq c_{7}, \\
\mathbb{E}\left(\left\|\mu_{n}\right\|_{\gamma}\right) \leq c_{8},
\end{gathered}
$$

where $\left\|\mu_{n}\right\|=\mu_{n}([\epsilon, T])$ denotes the total mass of $\mu_{n}$, then there is a subsequence of $\left\{\mu_{n}\right\}$, say $\left\{\mu_{n_{k}}\right\}$, such that $\mu_{n_{k}} \rightarrow \mu$ in $\mathcal{M}_{\gamma}^{+}$and $\mu$ is strictly positive with probability $\geq c_{6}^{2} /\left(2 c_{7}\right)$. In this case, it follows from (56) that $\mu$ has its support in $L_{x}^{\epsilon}$ almost surely. Moreover, (58) and the monotone convergence theorem together imply that the $\gamma$-energy of $\mu$ is finite. Hence Frostman's 
theorem yields (54) with $c_{5}=c_{6}^{2} /\left(2 c_{7}\right)$.

It remains to verify (57) and (58). By Fubini's theorem we have

$$
\begin{aligned}
\mathbb{E}\left(\left\|\mu_{n}\right\|\right) & =\int_{\epsilon}^{T} \int_{\mathbb{R}} \exp (-i \xi x) \exp \left(-\xi^{2} / 2\right) \mathbb{E}\left(\exp \left(i \xi S^{H}(t)\right)\right) d \xi d t \\
& =\int_{\epsilon}^{T} \int_{\mathbb{R}} \exp (-i \xi x) \exp \left(-\left(1+\sigma^{2}(t)\right) \xi^{2} / 2\right) d \xi d t \\
& =\int_{\epsilon}^{T} \sqrt{2 \pi /\left(1+\sigma^{2}(t)\right)} \exp \left(-x^{2} /\left(2\left(1+\sigma^{2}(t)\right)\right)\right) d t \\
& \geq \int_{\epsilon}^{T} \sqrt{2 \pi /\left(1+\sigma^{2}(t)\right)} \exp \left(-x^{2} / 2 \sigma^{2}(t)\right) d t:=c_{6} .
\end{aligned}
$$

Denote by $I_{2}$ the identity matrix of order 2 and $\operatorname{Cov}\left(S^{H}(s), S^{H}(t)\right)$ the covariance matrix of the random vector $\left(S^{H}(s), S^{H}(t)\right)$. Let $\Gamma=I_{2}+\operatorname{Cov}\left(S^{H}(s), S^{H}(t)\right)$ and $(\xi, \eta)^{\prime}$ be the transpose of the row vector $(\xi, \eta)$. Then

$$
\begin{aligned}
\mathbb{E}\left(\left\|\mu_{n}\right\|^{2}\right) & =\int_{\epsilon}^{T} \int_{\epsilon}^{T} \int_{\mathbb{R}} \int_{\mathbb{R}} \exp (-i(\xi+\eta) x) \exp \left(-(\xi, \eta) \Gamma(\xi, \eta)^{\prime} / 2\right) d \xi d \eta d s d t \\
& =\int_{\epsilon}^{T} \int_{\epsilon}^{T}(2 \pi / \sqrt{\operatorname{det} \Gamma}) \exp \left(-(x, x) \Gamma^{-1}(x, x)^{\prime} / 2\right) d s d t \\
& \leq \int_{\epsilon}^{T} \int_{\epsilon}^{T} 2 \pi / \sqrt{\operatorname{det} \operatorname{Cov}\left(S^{H}(s), S^{H}(t)\right)} d s d t .
\end{aligned}
$$

Since

$$
\operatorname{det} \operatorname{Cov}\left(S^{H}(s), S^{H}(t)\right)=\operatorname{Var}\left(S^{H}(s)\right) \operatorname{Var}\left(S^{H}(t) \mid S^{H}(s)\right),
$$

from conditions (77) and (44) we get: for all $s, t \in[\epsilon, T]$,

$$
\operatorname{det} \operatorname{Cov}\left(S^{H}(s), S^{H}(t)\right) \geq c_{9}|s-t|^{2 H_{i_{0}}} .
$$

where $c_{9}$ denotes a positive constant. Combining (61) and (62) we obtain:

$$
\mathbb{E}\left(\left\|\mu_{n}\right\|^{2}\right) \leq c_{10} \int_{\epsilon}^{T} \int_{\epsilon}^{T} 2 \pi /|s-t|^{H_{i_{0}}} d s d t .
$$

where $c_{10}$ denotes a positive constant. Since $\left.H_{i_{0}} \in\right] 0,1$, the last integral is a finite constant, and consequently

$$
\mathbb{E}\left(\left\|\mu_{n}\right\|^{2}\right) \leq c_{7}
$$

with $c_{7}=c_{10} \int_{\epsilon}^{T} \int_{\epsilon}^{T} 2 \pi /|s-t|^{H_{i_{0}}} d s d t$. 
Similar to (60), we have

$$
\begin{aligned}
& \mathbb{E}\left(\left\|\mu_{n}\right\|_{\gamma}\right) \\
= & \int_{\epsilon}^{T} \int_{\epsilon}^{T}|s-t|^{-\gamma} d s d t \int_{\mathbb{R}} \int_{\mathbb{R}} \exp (-i(\xi+\eta) x) \exp \left(-(\xi, \eta) \Gamma(\xi, \eta)^{\prime} / 2\right) d \xi d \eta \\
\leq & c_{11} \int_{\epsilon}^{T} \int_{\epsilon}^{T} 2 \pi|s-t|^{-\gamma-H_{i_{0}}} d s d t
\end{aligned}
$$

where $c_{11}$ denotes a positive constant. Since $\left.-\gamma-H_{i_{0}}=-1+H_{i_{0}} \delta \in\right]-1,0[$, we get $\mathbb{E}\left(\left\|\mu_{n}\right\|_{\gamma}\right)<c_{8}$ with

$$
c_{8}=c_{11} \int_{\epsilon}^{T} \int_{\epsilon}^{T} 2 \pi|s-t|^{-\gamma-H_{i_{0}}} d s d t,
$$

which completes the proof

\section{Study of the semimartingale property}

In this section, we will discuss for which values of the Hurst parameter $H$, $S^{H}(N, a)$ is a semimartingale. Let us first specify that in this paper, for a stochastic process $\left\{X_{t}, 0 \leq t \leq T\right\}$, we denote by $\mathcal{F}^{X}=\left(\mathcal{F}_{t}^{X}\right)_{0 \leq t \leq T}$ the own filtration of $X$, and we call $X$ a semimartingale if it is a semimartingale with respect to $\overline{\mathcal{F}}^{X}$, the smallest filtration that contains $\mathcal{F}^{X}$ and satisfies the usual assumptions. Let us start our study by the following lemma.

Lemma 19 Let us denote, for every $t>0$, by $\left\langle S^{H}(N, a)\right\rangle_{t} \quad$ [respectively $\left.V\left(S^{H}\right)_{t}\right]$ the quadratic variation [respectively the variation] of the process $S^{H}(N, a)$ on the interval $[0, t]$, and let us recall that $H_{i_{0}}$ denotes the parameter defined by (25). For every $t>0$,

1. if $H_{i_{0}}<1 / 2$ then, $<S^{H}(N, a)>_{t} \stackrel{a . s .}{=}+\infty$,

2. if, $H_{i}>1 / 2$ for every $i \in\{1, \ldots, N\}$, then

$$
<S^{H}(N, a)>_{t} \stackrel{a . s .}{=} 0 \text { and } V\left(S^{H}\right)_{t} \stackrel{a . s .}{=} \infty .
$$

Proof. 1. For any $n \in \mathbb{N} \backslash\{0\}, p \in \mathbb{N} \backslash\{0\}$ and $t>0$, we denote

$$
A_{n, p}=\sum_{j=1}^{n}\left|S_{\frac{j t}{n}}^{H}(N, a)-S_{\frac{(j-1) t}{n}}^{H}(N, a)\right|^{p},
$$

Assume that $<S^{H}(N, a)>_{t}<\infty$ a.s. Then $A_{n, 2} \rightarrow<S^{H}(N, a)>_{t}$ in probability as $n \rightarrow \infty$, so there is a subsequence $\left(n_{k}\right)$ such that 
$A_{n_{k}, 2} \rightarrow<S^{H}(N, a)>_{t}$ a.s. as $k \rightarrow \infty$ and therefore $\sup _{k} A_{n_{k}, 2}<\infty$ a.s. Let

$$
v(x)=\left(\sup _{k} \sum_{j=1}^{n_{k}}\left[x\left(\frac{j t}{n_{k}}\right)-x\left(\frac{(j-1) t}{n_{k}}\right)\right]^{2}\right)^{1 / 2}, x \in C([0, t]),
$$

$v$ is a measurable seminorm on $C([0, t])$ such that $v\left(S^{H}(N, a)\right)<\infty$ a.s. Then by Fernique's theorem (cf. 10]) $\mathbb{E}\left(v\left(S^{H}(N, a)\right)\right)^{q}<\infty$ for all $q>0$. Pick $p>2$ such that $H_{i_{0}}<\frac{1}{p}<\frac{1}{2}$. Then

$$
\begin{aligned}
& \mathbb{E}\left(A_{n_{k}, p}\right) \leq \mathbb{E}\left(\max _{j \leq n}\left|S_{j / n_{k}}^{H}(N, a)-S_{(j-1) / n_{k}}^{H}(N, a)\right|^{p-2} v\left(S^{H}(N, a)\right)^{2}\right) \\
\leq & {\left[\mathbb{E}\left(\max _{j \leq n}\left|S_{j / n_{k}}^{H}(N, a)-S_{(j-1) / n_{k}}^{H}(N, a)\right|^{2(p-2)}\right)\right]^{1 / 2}\left[\mathbb{E}\left(v\left(S^{H}(N, a)\right)^{4}\right)\right]^{1 / 2} . }
\end{aligned}
$$

Since $p>2$, the last expression tends to 0 as $k \rightarrow \infty$; in fact, by continuity of $S^{H}(N, a)$, Lebesgue's theorem and the fact that

$$
\mathbb{E}\left(\sup _{s \leq t}\left|S_{s}^{H}(N, a)\right|^{2(p-2)}\right)<\infty
$$

( again by Fernique's theorem with the seminorm $x \longmapsto \sup _{s \leq t}|x(s)|$ on $C([0, t])$ ), we have

$$
\mathbb{E}\left(\max _{j \leq n}\left|S_{j / n_{k}}^{H}(N, a)-S_{(j-1) / n_{k}}^{H}(N, a)\right|^{2(p-2)}\right) \rightarrow 0 \text { as } k \rightarrow \infty .
$$

But, by (15) and since the increments of $S^{H}(N, a)$ are Gaussian, it is easy to get

$$
a n^{1-p H_{i_{0}}} \leq E\left(A_{n, p}\right)
$$

where $a$ is a positive constant, which depends on $a_{i_{0}}, H_{i_{0}}, p$ and $t$. Since $1-p H_{i_{0}}>0$, we deduce that $\mathbb{E}\left(A_{n, p}\right)$ tends to $\infty$ as $n \rightarrow \infty$. Hence it is not possible that $<S^{H}(N, a)>_{t}$ be finite a.s. and by the $0-1$ law $\left\langle S^{H}(N, a)>_{t}=\right.$ $\infty$ a.s.

2. To check the first assertion, let us consider a sequence

$$
\left\{\tau_{n}: 0=t_{0}<t_{1}<\ldots<t_{n}=t,\right\}_{n \in \mathbb{N} \backslash\{0\}}
$$

of finite partitions of $[0, t]$ such that its mesh $\left|\tau_{n}\right|=\max _{i=1}^{n}\left|t_{i}-t_{i-1}\right| \stackrel{n \rightarrow \infty}{\longrightarrow} 0$. From Proposition $[5$ for every $\sigma, s \in[0, t] ; s \leq \sigma$ we have

$$
\sum_{i=1}^{N} a_{i}^{2} \gamma_{i}(\sigma-s)^{2 H_{i}} \leq E\left(S_{\sigma}^{H}(N, a)-S_{s}^{H}(N, a)\right)^{2} \leq \sum_{i=1}^{N} a_{i}^{2} \nu_{i}(\sigma-s)^{2 H_{i}}
$$


and consequently,

$$
C_{1}(\sigma-s)^{2 H_{i_{0}}} \leq E\left(S_{\sigma}^{H}(N, a)-S_{s}^{H}(N, a)\right)^{2} \leq C_{2}(\sigma-s)^{2 H_{i_{0}}}
$$

where

$$
C_{1}=a_{i_{0}}^{2} \gamma_{i_{0}} \quad \text { and } \quad C_{2}=\sum_{i=1}^{N} a_{i}^{2} \nu_{i} t^{2\left(H_{i}-H_{i_{0}}\right)} .
$$

For any integer $n \geq 1$, denote

$$
\Delta_{t}^{\tau_{n}}=\sum_{j=1}^{n}\left(S_{t_{j}}^{H}(N, a)-S_{t_{j-1}}^{H}(N, a)\right)^{2} .
$$

From equation (68), we have

$$
\begin{aligned}
E\left(\Delta_{t}^{\tau_{n}}\right) & \leq C_{2} \sum_{j=1}^{n}\left(t_{j}-t_{j-1}\right)^{2 H_{i_{0}}} \\
& \leq C_{2}\left|\tau_{n}\right|^{2 H_{i_{0}}-1} \sum_{j=1}^{n}\left(t_{j}-t_{j-1}\right) \\
& =C_{2}\left|\tau_{n}\right|^{2 H_{i_{0}}-1} t .
\end{aligned}
$$

Since $\lim _{n \rightarrow \infty} C_{2}\left|\tau_{n}\right|^{2 H_{i_{0}}-1} t=0, \quad \lim _{n \rightarrow \infty} E\left(\Delta_{t}^{\tau_{n}}\right)=0$.

Hence the sequence $\left(\Delta_{t}^{\tau_{n}}\right)$ converges to 0 in probability, which yields that

$$
<S^{H}(N, a)>_{t}=0 \text { a.s. }
$$

In order to get the second assertion in 2., it suffices to follow the same procedure as that of the proof of 1. in Lemma 19, and to use Fernique's Theorem and equation (15).

As a consequence of lemma 19 we get:

Corollary 20 If it exists $i \in\{1, \ldots, N\} ; H_{i}<1 / 2$ and $a_{i} \neq 0$, or if $H_{i}>1 / 2$, for every $i \in\{1, \ldots, N\}$, then the $m s f B m S^{H}(N, a)$ is not a semimartingale.

Proof. In the case where it exists $i \in\{1, \ldots, N\} ; H_{i}<1 / 2$ and $a_{i} \neq 0$, the corollary is a direct consequence of the assertion 1. of Lemma 19. So let us check the second case. Suppose $S^{H}(N, a)$ is a right-continuous semimartingale. Hence, $S^{H}(N, a)$ can be written in the form

$$
S^{H}(N, a)=M_{t}^{H}+A_{t}^{H}
$$

where $M_{0}^{H}=A_{0}^{H}=0, M^{H}$ is an a.s. right-continuous local martingale with respect to $\overline{\mathcal{F}}^{S^{H}(N, a)}$ and $A^{H}$ an a.s. right-continuous, $\overline{\mathcal{F}}^{S^{H}}$ - adapted finite 
variation process. It follows, by Theorem II.22 of [15] and Lemma 19, that, for every $t \in[0 ; 1]$,

$$
0=<S^{H}(N, a), S^{H}(N, a)>_{t}=<M^{H}, M^{H}>_{t} .
$$

By Theorem $I I .27$ of [15], $M^{H}$ is itself a zero process; and hence $S^{H}(N, a)=A^{H}$ has finite variation. This contradicts assertion 2. of Lemma 19.

In the following lemma, we treat the case where it exists $k_{0} \in\{1, . ., N\} ; H_{k_{0}}=$ $1 / 2$ such that $a_{k_{0}} \neq 0$ and for every $\left.i \in\{1, \ldots, N\} \backslash\left\{k_{0}\right\}, H_{i} \in\{1 / 2\} \cup\right] 3 / 4,1[$.

Lemma 21 For every $T>0$, if it exists $k_{0} \in\{1, . ., N\} ; H_{k_{0}}=1 / 2 ; a_{k_{0}} \neq 0$ and for every $\left.i \in\{1, \ldots, N\} \backslash\left\{k_{0}\right\}, H_{i} \in\{1 / 2\} \cup\right] 3 / 4,1[$, the process

$$
S^{H}(N, a)=\left\{S_{t}^{H}(N, a), t \in[0, T]\right\}
$$

is, in its own filtration, a semimartingale equivalent in law with $a_{k_{0}}$ times a Brownian motion.

Proof. The process $S^{H}(N, a)$ can be written $S^{H}(N, a)=a_{k_{0}}\left(\xi^{1 / 2}+X_{t}\right)$ where

$$
X_{t}=\sum_{\substack{i=1 \\ i \neq k_{0}}}^{N} \frac{a_{i}}{a_{k_{0}}} \xi^{H_{i}}(t) .
$$

The process $X_{t}$ is Gaussian and its covariance function

$$
R(s, t)=\sum_{\substack{i=1 \\ i \neq k_{0}}}^{N} \frac{a_{i}^{2}}{a_{k_{0}^{2}}}\left(t^{2 H_{i}}+s^{2 H_{i}}-1 / 2\left[|s+t|^{2 H_{i}}+|t-s|^{2 H_{i}}\right]\right),
$$

is positive definite, twice continuously differentiable on $[0, T]^{2} \backslash\{(s, t) ; t=s\}$ and for every $(s, t) \in[0, T]^{2} \backslash\{(s, t) ; t=s\}$,

$$
\frac{\partial^{2} R(s, t)}{\partial s \partial t}=\sum_{\substack{i=1 \\ i \neq k_{0}}}^{N} \frac{a_{i}^{2}}{a_{k_{0}^{2}}} H_{i}\left(2 H_{i}-1\right)\left[|t-s|^{2 H_{i}-2}-|s+t|^{2 H_{i}-2}\right] .
$$

So, because $\left.H_{i} \in\{1 / 2\} \cup\right] 3 / 4 ; 1\left[\right.$, for every $i \in\{1, \ldots, N\} \backslash\left\{k_{0}\right\}$, it's easy to check that $\frac{\partial^{2} R}{\partial s \partial t} \in L^{2}\left([0, T]^{2}\right)$.

On the other hand, $\xi^{1 / 2}$ is a Brownian motion independent of $X_{t}$. Consequently, according to [3], the process

$$
\left\{\xi_{t}^{1 / 2}+X_{t}, t \in[0, T]\right\}
$$


is, in its own filtration, a semimartingale equivalent in law to a Brownian motion and the lemma is proved.

We finish this section by the study of the last case. That is, the case where it exists $k_{0} \in\{1, \ldots, N\} ; H_{k_{0}}=1 / 2, H_{i} \geq 1 / 2$ for every $i \in\{1, \ldots, N\} \backslash\left\{k_{0}\right\}$,

and it exists $\left.\left.i \neq k_{0} ; H_{i} \in\right] 1 / 2,3 / 4\right]$. We study a more general case, where, for every $k \in\{1, \ldots, N\}, H_{k} \geq 1 / 2$ and it exists $\left.\left.i \in\{1, \ldots, N\} ; H_{i} \in\right] 1 / 2,3 / 4\right]$. Let us first recall the definition of a quasimartingale.

Definition: A stochastic process $\left(X_{t}\right)_{t \geq 0}$ is a quasimartingale if for every $T>0, X_{t} \in L^{1}$ for all $t \in[0, T]$, and

$$
\sup _{\tau} \sum_{j=0}^{k-1}\left\|E\left(X_{t_{j+1}}-X_{t_{j}} \mid \mathcal{F}_{t_{j}}^{X}\right)\right\|_{1}<\infty
$$

where $\tau$ is the set of all finite partitions

$$
0=t_{0}<t_{1}<\ldots<t_{n}=\text { T of }[0, T] .
$$

In the following key lemma we specify the relation between quasimartingale and semimartingale in the case of our process $S^{H}(N, a)$.

Lemma 22 If $S^{H}(N, a)$ is not a quasimartingale, it is not a semimartingale, with respect to its own filtration.

Proof. The proof is similar to that of Lemma 4.2 in [5]; in fact, only Gaussianity was used there.

Lemma 23 If, for all $k \in\{1, \ldots, N\}, H_{k} \geq 1 / 2$, and it exists $k_{0} \in\{1, \ldots, N\}$; $1 / 2<H_{k_{0}} \leq 3 / 4$ and $a_{k_{0}} \neq 0$, then $S^{H}(N, a)$ is not a quasimartingale, and in particular, it's not a semimartingale.

Proof. The proof is an easy extention of the proof of Lemma 25 in [8] to our general case.

\section{References}

[1] R.J. Adler, The Geometry of Random Fields. John Wiley \& Sons Ltd., New York.

[2] A. Ayache and Y. Xiao, Asymptotic properties and Hausdorff dimensions of fractional Brownian sheets. J. Fourier Anal. Appl. 11,407 - 439. 
[3] F. Baudoin and D. Nualart, Equivalence of Volterra processes. Stochastic Processes and their Applications. 107(2), 327 - 350.

[4] T. Bojdeckia, L.G. Gorostizab and A. Talarczyka, Sub-fractional Brownian motion and its relation to occupation times. Statistics \& Probability Letters Volume 69, Issue 4, 1 October 2004, Pages $405-419$.

[5] P. Cheridito, Mixed Fractional Brownian Motion Bernoulli 7(2001); 913..934.

[6] C. Dellacherie and P.A. Meyer, Probabilités et Potentiel : Chapitres $V \grave{a}$ VIII, Paris, Hermann, 1980.

[7] C. Doleans-Dade and P.A. Meyer, Integrales stochastiques par rapport aux martingales locales. Seminaire Proba. IV. Lecture Notes in Mathematics (1970). 124, $77-107$.

[8] C. El-Nouty and M. Zili, On The Sub-Mixed Fractional Brownian Motion . arXiv:1206.4291v1 [math.PR] 19 Jun 2012.

[9] K. Falconer, Fractal Geometry. Mathematical foundation and applications. John Wiley and Sons, Chichester, 1990.

[10] X. Fernique, Intégrabilité des vecteurs Gaussiens. C.R. Acad. Sci. Paris Sér. A, 270 (25), 1698-1699.

[11] G.H. Golub, C.F. Van Loan, Matrix Computations. Hopkins University Press (1989).

[12] J.P. Kahane, Some Random Series of Functions. 2nd edition. Cambridge University Press, Cambridge, UK.

[13] B. B. Mandelbrot, J.W. Van Ness Fractional Brownian Motions, Fractional Noises and Applications. SIAM Review, vol.10, No 4, October 1968.

[14] Y. Miao, W. Ren, Z. Ren, On the Fractional Mixed Fractional Brownian Motion. Applied Mathematical Sciences, Vol. 2, 2008, no. 35, 1729 - 1738.

[15] P. Protter, Stochastic Integration and Differential Equations. SprigerVerlag 2009.

[16] D. Revuz, M.Yor Continuous martingales and Brownian motion. Grundlehren der mathematischen Wissenschaften 293, Springer.

[17] C. Stricker, Quelques remarques sur les semimartingales Gaussiennes et le problème de l'innovation, In H. Korezlioglu, G. Mazziotto and J. Szpirglas (eds), Filtering and Control of Random Processes, Lecture Notes in Control and Inform. Sci. 61, 260-276. Berlin: Springer-Verlag.

[18] M. Talagrand, Hausorff measure of trajectories of multiparameter fractional Brownian motion. The annals of Probability. 1995, Vol. 23, No. 2, 767-775. 
[19] F. Testard, Polarité, points multiples et géométrie de certain processus Gaussiens. Publ. du Laboratoire de Statistique et Probabilités de l'U.P.S. Toulouse, $01-86$.

[20] C. Thäle, Further Remarks on Mixed Fractional Brownian Motion. Applied Mathematical Sciences, Vol. 3,2009, no. 38, 1885 - 1901.

[21] C. Tudor, Some properties of the sub-fractional Brownian motion. Stochastics. Vol. 79, No.5, October 2007, $431-448$.

[22] D. Wu and Y. Xiao, On local times of antisotropic Gaussian random fields. Communications on Stochastic Analysis. Vol. 5, No. 1(2011)15 - 39.

[23] Y. Xiao, Dimension results for Gaussian vector fields and index- $\alpha$ stable fields. The annals of Probability. 1995, Vol. 23, No. 1, $273-291$.

[24] Y. Xiao, Sample path properties of anisotropic Gaussian random fields. A Minicourse on Stochastic Partial Differential Equations, Khoshnevisan, D., Rassoul-Agha, F. (Eds.), pp 145(212), Springer, New York, 2009.

[25] L. Yan and G. Shen, On the collision local time of sub-fractional Brownian motions. Statistics and Probability Letters 80 (2010) $296-308$.

[26] M. Zili, On the Mixed- Fractional Brownian Motion, Journal of Applied Mathematics and Stochastic Analysis Volume 2006 (2006), Article ID 32435.

\section{Author information}

Mounir Zili, Department of Mathematics, Faculty of Sciences of Monastir, Avenue de l'environnement, 5019 Monastir, Tunisia

Mounir.Zili@fsm.rnu.tn 